\title{
Structural OPTIMiZation OF SPACE COMPONENTS ADAPTED FOR 3D PRINTING
}

\author{
Camelia Elena Munteanu, Alexandru-Mihai Cismilianu, \\ Alina-Ioana Chira \& Daniela Baran
}
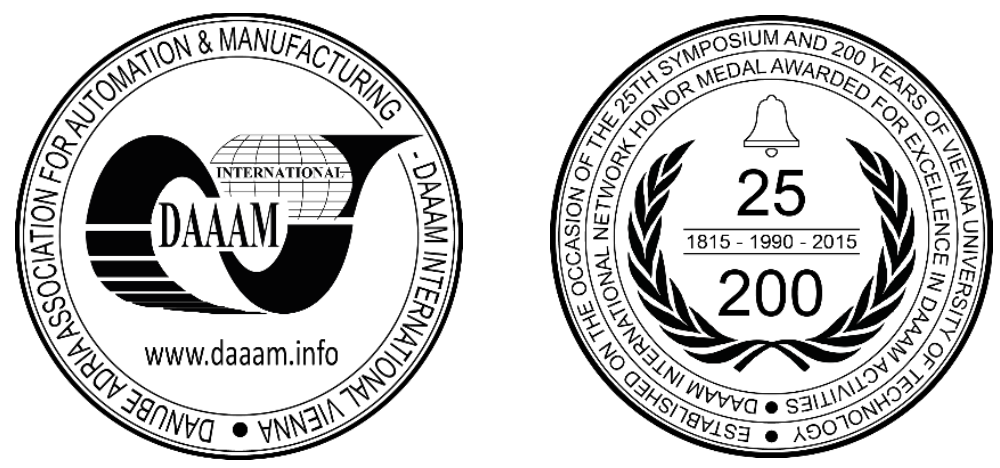

This Publication has to be referred as: Munteanu, C[amelia] E[lena]; Cismilianu, A[lexandru] -[ Mihai]; Chira, A[linaIoana] \& Baran, D[aniela] (2017). Structural Optimization of Space Components Adapted for 3D Printing, Proceedings of the 28th DAAAM International Symposium, pp.0821-0825, B. Katalinic (Ed.), Published by DAAAM International, ISBN 978-3-902734-11-2, ISSN 1726-9679, Vienna, Austria

DOI: $10.2507 / 28$ th.daaam.proceedings. 115

\begin{abstract}
In the space sector reducing the cost through innovative designs, can be achieved through mass reduction and shorter development time. Additive layer manufacturing (ALM) is a process which enables, not only a new cycle of optimization in terms of mass and performance, but also a minimum lead time for small series production of complex parts.

The objective of this paper is to design a part as light as possible while respecting the specific requirements. Specialized optimization tools were used in order to significantly reduce the number of design iterations. In order to cut down the mass furthermore, while increasing the stiffness, a hollow structure was considered. Internal cavities may raise a problem of powder evacuation in case of powder fusion processes. Concerning contamination problems of the spacecraft's (S/C) components, a powder removal procedure was closely developed with the manufacturer. As an outcome, a lighter space component with a lower production cost and fewer points of potential failure was obtained.
\end{abstract}

Keywords: Structural optimization; 3D Printing; internal cavities; powder evacuation; space applications

\section{Introduction}

The quest for light and stiff structures by industries such as the medical industry, the aerospace industry and other industries drives the progress in the additive manufacturing technology[1].

Cost reducing has become the focus of most researches and activities developed in the space sector. Although it is extremely expensive to manufacture and launch a space vehicle, the most expensive part is the development of all the sub-processes and the optimization of all the components. An important approach of cost cutback is weight reduction and shorter development time.

ALM is an emerging manufacturing technology which provides the potential for significant weight savings through optimization due to the relatively relaxed design constraints imposed. The part cost for ALM is independent of complexity, and so there is a 'virtuous circle' whereby weight savings through optimization also result in cost savings as the amount of material used to make the part reduces.[2]

The process of building an object by layering material instead of subtracting it from a larger block of material is often cited as having the potential to revolutionize the manufacturing industry. 
Aerospace and Space industry has taken a leading role in the development, implementation and industrialization of ALM. The main benefits of the ALM process are design flexibility, low material waste and low cost of producing parts from hard materials that are otherwise difficult to manufacture [3].

ALM is known to provide more design freedom than conventional manufacturing methods, which encourages the implementation of numerical optimization methods in the design process in order to reduce weight by eliminating unneeded material [4]. Topology optimization offers a faster way to create load specific structures thus, enabling the industry to unlock enormous lightweight design potential by using a powerful design tool in combination with the use of ALM.

Using topology optimization, the entire structure can be modified. This type of analysis reduces drastically the number of exchanges between the design and stress departments which leads to a downsize in time and cost.

The key to realizing metal parts using ALM is in understanding that 3D printed metal parts differ in properties from machined ones just like aluminium cast parts differ from aluminium machined parts.

\section{Input}

The aim of the project was to demonstrate the potential weight savings achievable using the design freedom offered by ALM, while respecting the conditions imposed by the customer.

The utility of an optimization software is presented by determining the optimum material distribution of a support bracket for space use. For the design of the bracket the following input data was set (Fig. 1.). In the input data figure there are two groups of thrusters disposed in the front of the envelope. We also have the attachment points which are disposed in the same plane.

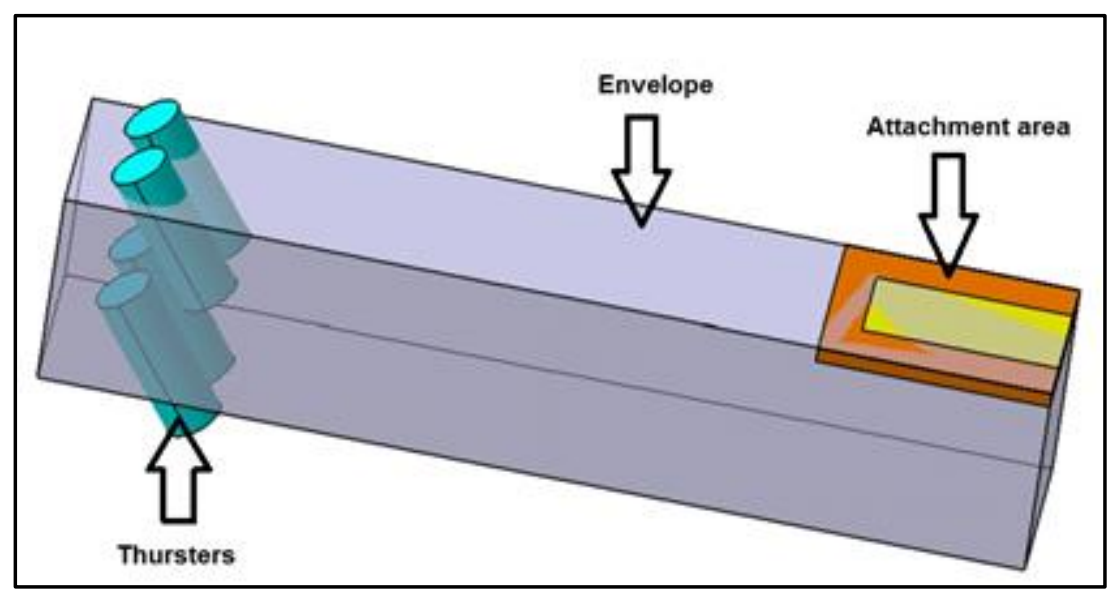

Fig. 1. Envelope \& boundary conditions

The main requirements are to develop a bracket which can hold the thrusters in the given positions and fit the envelope. A first frequency value of $90 \mathrm{~Hz}$ or above has to be provided and the resulted structure must withstand a $30 \mathrm{~g}$ load on all principal directions (X, Y, and Z). The aim is to obtain a structure by minimizing the mass while maximizing the frequency with respect of the constraints. Eight attachment points on the orange area, were considered to be enough. The thruster attachment points to the structure was developed closely with the customer.

\section{Design approach}

By carefully analysing the given input, eight attachment points on the orange area, were considered to be enough. In order to generate a conceptual design, topological optimization is used. The software used to perform the analysis is INSPIRE [5].

The design space is presented in Fig. 2.a). The objective function considered was to minimize the weighted compliance. The result of the optimization analysis is presented in Fig. 2.b). It can be observed that are no internal elements and most of the material is distributed on the sides. An interesting and expected fact are the rounded shape of the elements that connect the last screws with the rest of the structure.

Using this structure, a new geometry presented in Fig. 2.c). was developed and used for a second iteration of optimization. In order to give more material and space for the optimization analysis, all the constructive elements were considered thicker. The upper and lower sides were not fully defined from the first analysis, so in those areas, for the second iteration of optimization, thin plates were considered. The second optimization offered a better understanding of the distribution of the material.

The result is presented in Fig. 2.d). The elements starting from the last screws are even more rounded. Also in the upper and lower part, some elements are significantly more visible. 


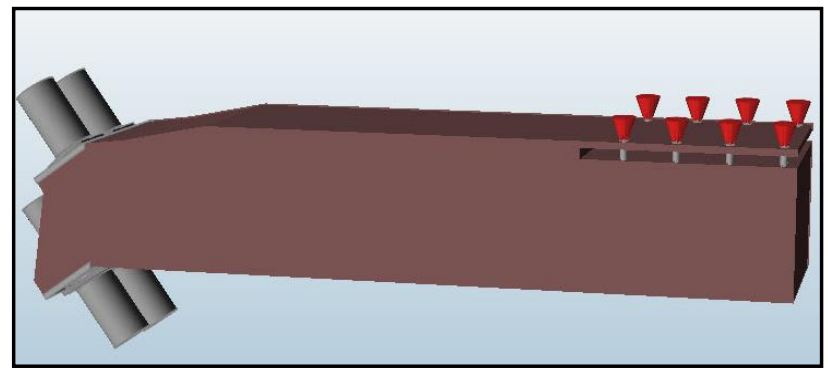

a) Input design space

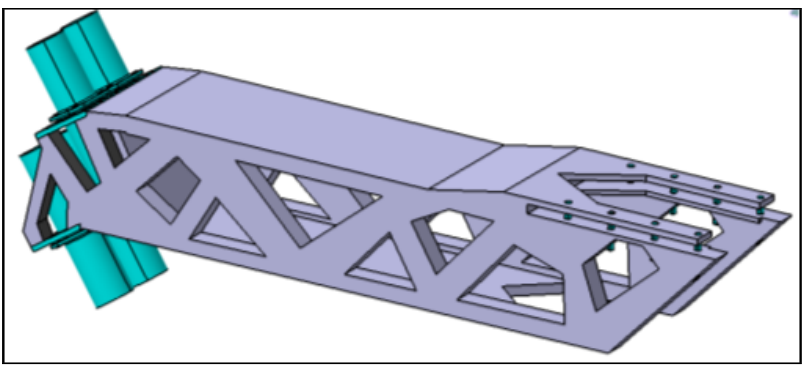

c) Second iteration design

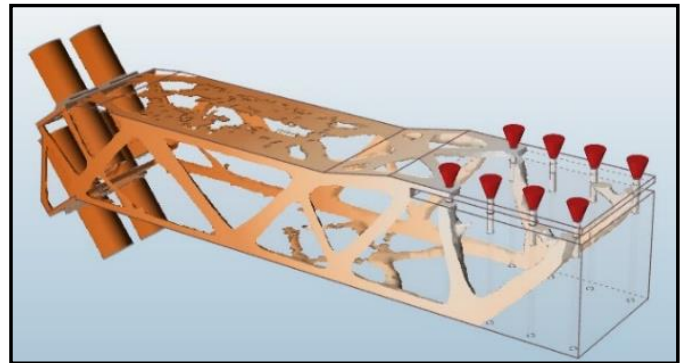

b) Optimization result

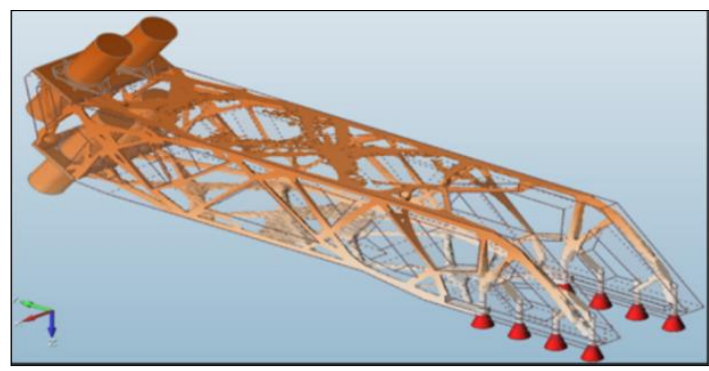

d) Second iteration of optimization

Fig. 2. Design iterations

\section{Final design and manufacturing}

By carefully analysing the distribution of the material given by the optimizer and respecting some conditions, like access in the interior of the bracket, ease of installation, providing connections with external elements, a new design was considered (Fig. 3.).

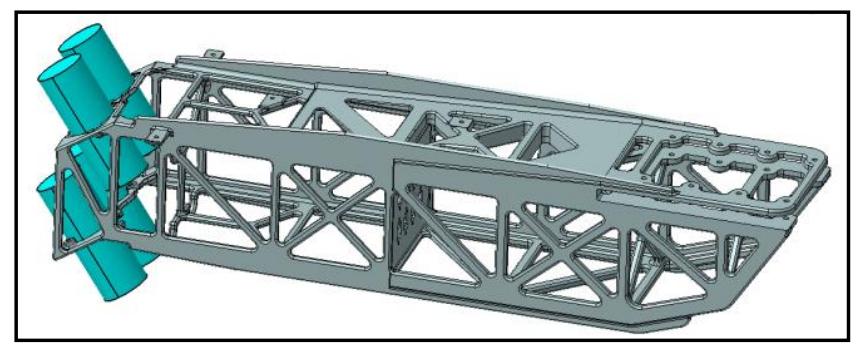

a) Second design reconstruction

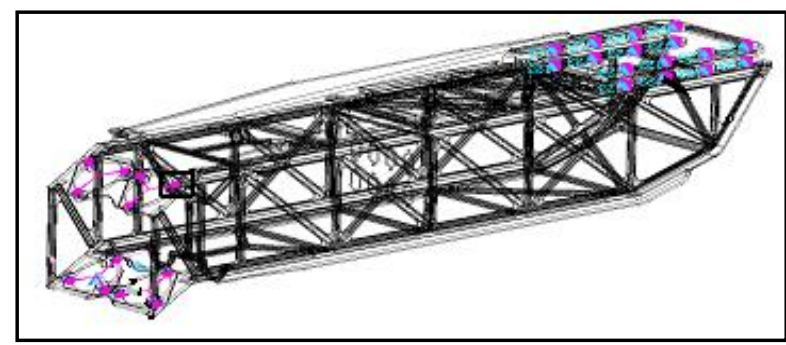

b) Internal cavities

Fig. 3. Final design

The aim was to provide a part as lightweight as possible while respecting a stiffness requirement of over $90 \mathrm{~Hz}$. Taking the advantages offered by ALM and its fewer restrictions regarding the geometry complexity, it was considered a part with internal cavities Fig. 3.b). which lead to a structure 30\% lighter.

The new design was analysed using finite element method and proven to withstand the loads considered and with the first frequency of $95 \mathrm{~Hz}$. Topology optimization allowed selecting the best elements in the given design space to maximize the use of material.

The next important step is manufacturing. As said before, ALM was chosen to manufacture the part. Although this process gives enormous shape freedom of the part, which can't be easily obtained with traditional manufacturing techniques, there are some aspects to consider when using this method. The parts that are intended to be made via ALM need to fit as a piece, or as many components in the largest printer available for the design team. For this article, Concept Laser Xline 1000R [6] was considered because of the large overall dimensions of the bracket.

For an evaluation of the structure, LAAM made the following estimation regarding the volume of the part, the estimated support volume and the estimated build time, presented in Fig. 4. 
- Machine: Xline1000r

- $\quad$ Build volume: $630 \times 400 \times 500$ $\mathrm{mm}$

- Material: AlSi7Mg0,6

- Volume: $672 \mathrm{~cm} 3$

- Support volume: $1100 \mathrm{~cm} 3$

- Time estimation: 10 days

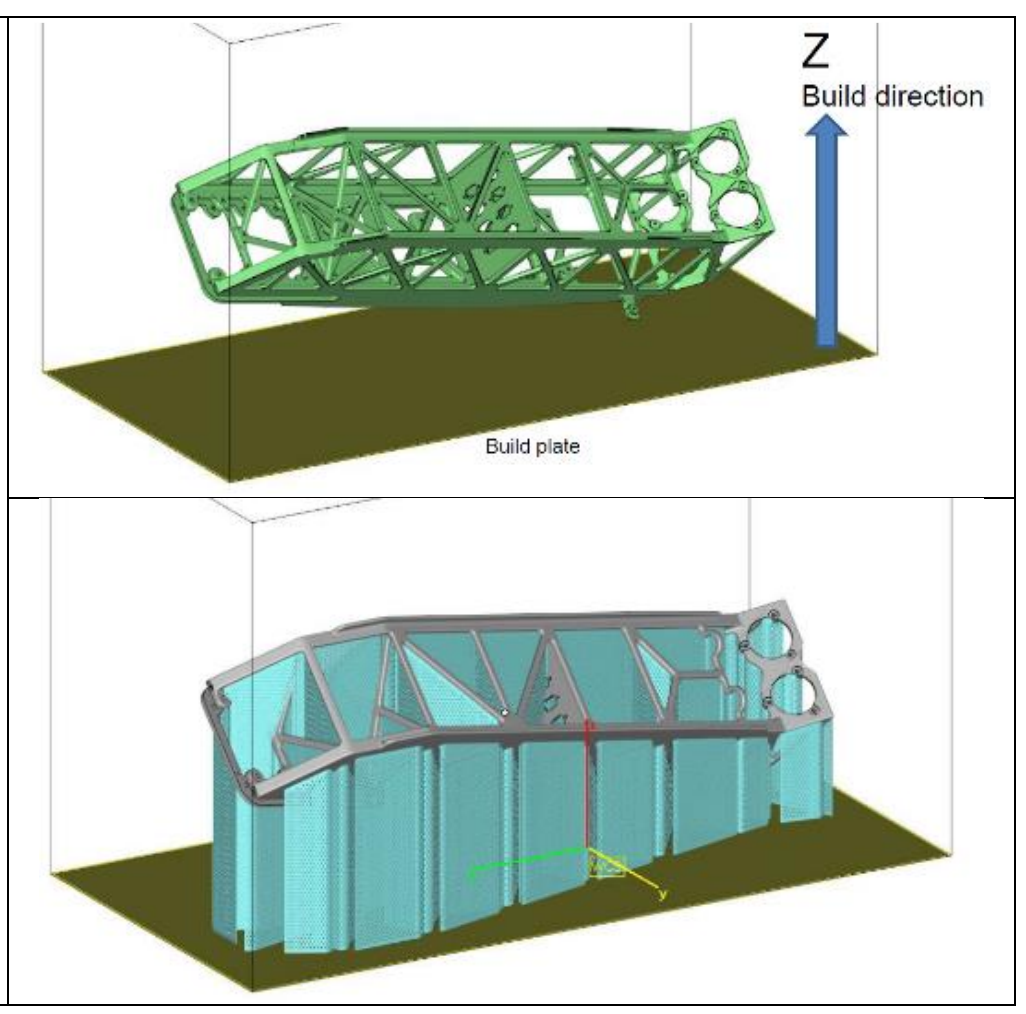

Fig. 4. Part orientation on build plate and support disposal courtesy of LAAM

As mentioned before, the bracket is intended to be manufactured hollow. For this purpose, evacuation holes are needed in order to safely evacuate all the powder.

Powder evacuation holes are directly dependent with the oriented part on the manufacturing build plate. The evacuation is intended to be made by shaking the part and by using pressured air. The powder must be evacuated before the elimination of supports, because if the support is removed without a heat treatment for stress relieve, the part will deform. If the heat treatment is made before the support removal there is a high risk that the powder will weld with the bracket making impossible the evacuation.
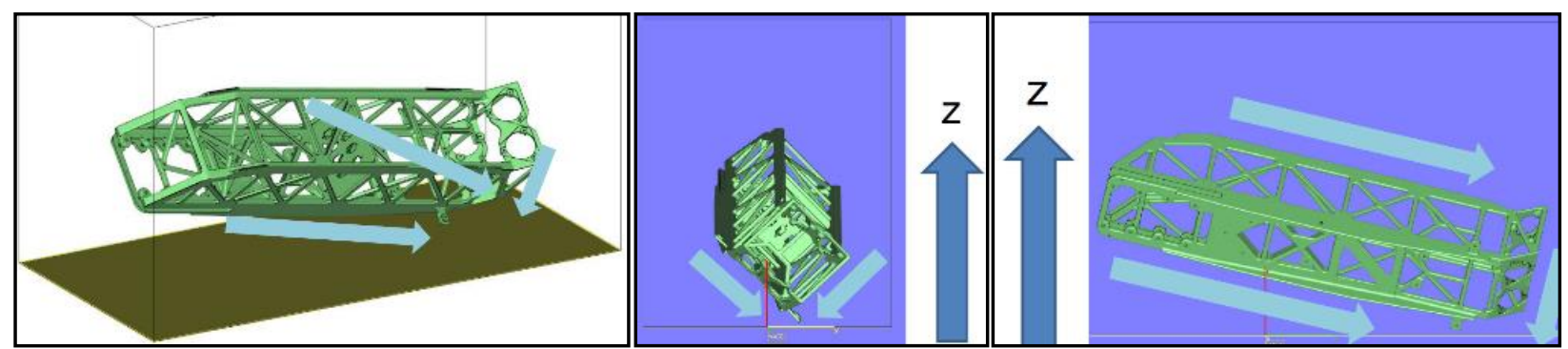

Fig. 5. Evacuation holes placement indications courtesy of LAAM

Before placing evacuation holes on the part, all the internal cavities must be interconnected, where possible, for an efficient removal of the powder. Where the cavities can't be interconnected, special evacuation holes must be made. All holes have to be placed then, as much as possible at the edge of the cavity taking in account the evacuation holes orientation directions presented in Fig. 5.

Taking into account the fact that the powder evacuation will be made by shaking the part and pressurized air, a powder flow circulation is presented. On the side, the cavities have evacuation holes only on the far ends (one of them represented in lower right of Fig. 6. The powder flow circulation is made because, if the red and blue pipe where connected as one, the pressurized air blown through the end will dissipate and the powder evacuation will be inefficient. 


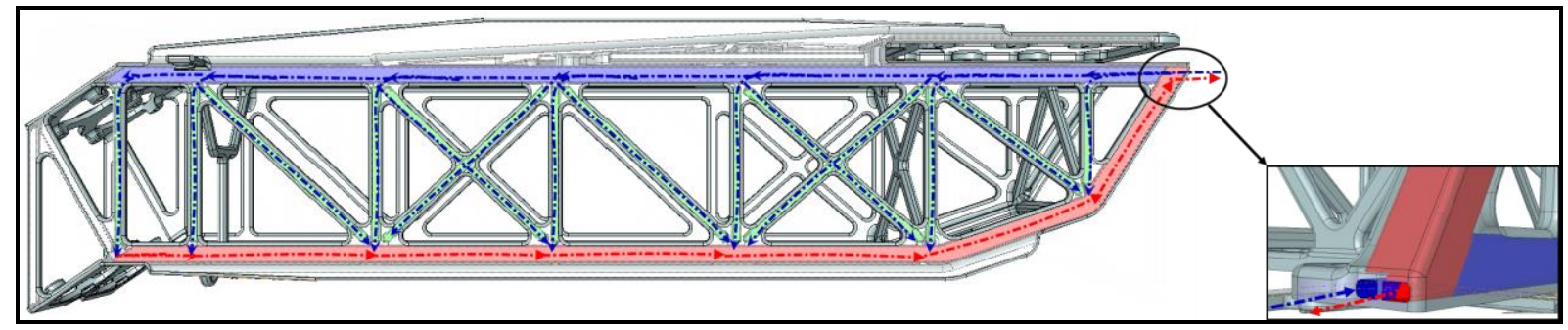

Fig. 6. Proposed powder-flow circulation

Because contaminating nearby components is a high-risk issue, and any remaining powder in the cavities prove to be a problem. For a designer, the essential question is how much contamination from all sources can be tolerated without causing a given spacecraft system to degrade below a critical performance level, or fail altogether [7]. In order to mitigate the risk CT scan has to be made in order to see if there is any remaining powder.

Choosing and properly implementing the best ALM process, material and post-processing combination for intended application is critical for success [8].

\section{Conclusions}

The aim of this paper was to obtain a part with an optimum distribution of material capable to withstand the loads applied while respecting the boundary conditions. Another important aspect was to provide access to some area inside the structure and to supply connections with external elements which imposed some limitation on the geometry obtained.

Using INSPIRE a load sufficient structure was obtained and analysed in a much shorter time of development. A detailed FEM analysis was performed only at the end of the process.

The support brackets evolution from the design space given as input to a final design ready to be printed was presented.

It was proven that INSPIRE is a powerful tool which if properly used, leads to advanced structures, with a dramatically reduced number of iterations between the design and stress departments. Being able to achieve faster an optimized structure enables a downsize in lead time and qualified personnel needed. Applying the steps presented in this paper to a larger scale can provide significant cost reductions.

\section{Acknowledgement}

The manufacturing part of the paper was possible through the know-how developed from the iterations that we had with LAAM - LISI AEROSPACE ADDITIVE MANUFACTURING Powered by POLY-SHAPE. We want to thank LAAM for providing us pictures for the manufacturing chapter. We also want to thank Elisabeth REY and Sébastien EYRIGNOUX for their cooperation.

\section{References}

[1] Hanzl, P[avel]; Zetek, M[iroslav] \& Zetkova, I[vana] (2016). Cellular Lattice Structure Produced by Selective Laser Melting and its Mechanical Properties, Proceedings of the 26th DAAAM International Symposium, pp.0748-0752, B. Katalinic (Ed.), Published by DAAAM International, ISBN 978-3-902734-07-5, ISSN 1726-9679, Vienna, Austria; DOI:10.2507/26th.daaam.proceedings.104

[2] Topology Optimisation of an Aerospace Part to be Produced by Additive Layer Manufacturing (ALM),Case Study, ALTAIR HyperWorks

[3] Maximizing the Potential of Additive Manufacturing with Design Optimization, Altair ProductDesign library of „Success Stories”, www.altairproductdesign.com

[4] Joona Seppälä, Andreas Hupfer, Topology Optimization in Structural Design of a LP Turbine Guide Vane: Potential of Additive Manufacturing for Weight Reduction, ASME Turbo Expo 2014: Turbine Technical Conference and Exposition, ISBN: 978-0-7918-4576-9

[5] http://www.solidthinking.com/Inspire2017.html

[6] https://www.concept-laser.de/en/products/machines.html

[7] Gary Pippin, Russ Crutcher, Spacecraft contamination issues from LDEF: Issues for design

[8] I. Gibson, D. W. Rosen, B. Stucker, Additive Manufacturing Technologies - Rapid Prototyping to Direct Digital Manufacturing, Springer Science+Bussiness Media, LLC 2010, ISBN 978-1-4419-1119-3 